\title{
Vaccine development/Prion disease/Viral-induced tumours
}

\author{
Chairpersons: R. Levy (Chicago, USA) \\ D. Riesner (Düsseldorf, D) \\ K. Khalili (Philadelphia, USA)
}

\author{
107 \\ Conditional virus replication as an approach \\ to a safe live-attenuated HIV-1 vaccine \\ B. Berkhout, K. Verhoef, G. Marzio, B. Klaver, M. Vink, \\ X. Zhou, A.T. Das \\ University of Amsterdam (Amsterdam, NL)
}

No safe and effective vaccine has been developed for the prophylaxis of AIDS. Live-attenuated viruses are the most effective vaccines tested thus far, but such variants are considered unsafe becaus e continued replication can lead to the selection of faster-replicating variants that are pathogenic (1). We therefore designed a novel vaccine strategy in which an HIV-1 virus is used that replicates exclusively in the presence of the non-toxic effector doxycycline (dox). This was achieved by replacement of the viral TAR-Tat system for transcriptional activation by the E. coli-derived Tetsystem for inducible gene expression (dox-regulated rtTA transcriptional trans-activator and the tetO DNA binding site). These designer 'HIV-rtTA' viruses replicate in a strictly dox-dependent manner both in a $\mathrm{T}$ cell line and in primary blood cells (2). Furthermore, the rate of replication can be fine-tuned by simple variation of the dox-concentration. These HIV-rtTA viruses may represent improved vaccine strains because their replication can be turned on and off at will. Moreover, we will present evidence that the Tetsystem is fine-tuned by spontaneous virus evolution to optimize its new function in virus replication in human cells (3). we identified rtTA protein variants with improved potency and increased dox-sensitivity. Thus, virus evolution can be exploited to select for novel Tet-systems with modified specificity and increased sensitivity. These reagents have proven very useful for optimization of our HIV-rtTA vaccine candidate. In addition, the modified reagents may be of particular use in other applications of the Tet-system, e.g. the regulation of transgene expression in transgenic animals and gene therapy vectors. For instance, a vector with a more sensitive Tet-system may allow gene therapy in the brain, where much lower dox-levels can be reached.

1. Berkhout, et al. (1999). J. Virology 73: 1138-1145.

2. Verhoef, et al. (2001). J. Virology 75: 979-987.

3. Marzio, et al. (2001). Proc. Natl. Acad. Sci. USA, 98: 63426347.

\section{8}

The immunobiology of prion diseases

A. Aguzzi

Institute of Neuropathology (Zurich, CH)

Mice deficient in the normal prion protein are resistant to exposure to prion infectivity, and expression of the normal prion protein by neurons is necessary for the development of histological damage. But how do prions reach the brain after entering the body from peripheral sites? The first portal of entry in the gut may be represented by M-cells. Neuroinvasion, i.e. the process by which prions march through the body of the host towards the brain, is dependent upon expression of the normal prion protein in a non-hematopoietic extracerebral site. We therefore developed the hypothesis that neuroinvasion takes place in two distinct steps: first the lymphoreticular system is diffusely colonized by the agent, while at a later time infectivity progresses from lymphoreticular organs to the central nervous system, probably via sympathetic nerves. There is an absolute requirement for B-lymphocytes in peripheral prion pathogenesis. Surprisingly, the presence of the normal prion protein is not necessary on B-lymphocytes to enable them to support this process. The mechanism of action of B lymphocyte may consist of presentation of lymphotoxin-B to follicular dendritic cells. This paves the way to post-exposure prophylaxis strategies that exploit the anti-prion effect of soluble lymphotoxin- $\beta$ receptors. Why do follicular dendritic cells accumulate prions? We tested the hypothesis that prion uptake may be complement-mediated. Indeed, certain components of the complement system (C1q, CR1/2) proved to play an important role in pathogenesis. Finally, we have found that transgenic expression of an anti-PrP antibody heavy chain suffices to confer to mice antiprion protection-a finding that may be relevant to the development of antiprion vaccines. 


\section{9}

\section{Effect of tricyclic compounds quinacrine and chlorpromazine on prion infectivity}

\section{Korth, S. Prusiner}

University of California (San Francisco, USA)

Permanently scrapie-infected mouse neuroblastoma (ScN2a) cells serve as a cellular model for prion disease and have been used for antiprion drug discovery. We recently described the tricyclic antiprion compounds as a new class of antiprion compound s characterized by a tricyclic scaffold with an aliphatic side-chain extending from the middle ring moiety. Treatment of ScN2a cells with these compound s led to disappearance of the pathogenic, disease-associated form of the prion protein (1). Here we describe the effects of two prominent members of this class of compounds, quinacrine and chlorpromazine, on prion infectivity of ScN2a cells. We treated ScN2a cells with different concentrations of quiancrine and chlorpromazine and inoculated lysates into transgenic mice overexpressing PrPC. Inoculation of cells treated with $0.8 \mu \mathrm{M}$ of quinacrine did not result in disease; at a quinacrine treatment concentration of $0.4 \mu \mathrm{M}$, cells retained infectivity, with two of five inoculated animals dying. Interestingly, treatment of ScN2a cells with chlorpromazine at doses greater than $5 \mu \mathrm{M}$ led to disappearance of the pathogenic, disease-associated PrPSc as observed by Western blotting; inoculation of mice with these cell lysates, however, showed that prion infectivity persisted in cells treated with $8 \mu \mathrm{M}$ chlorpromazine (four of five inoculated animals became ill). These results suggest that acridine-derivative quinacrine is superior to phenothiazine-derivative chlorpromazine in clearing prion infectivity and may thus be more promising for clinical trials for treating patients with Creutzfeldt-Jakob disease.

1. Korth C., May B.C.H., Cohen F.E., S.B. Prusiner. (2001). Acridine and phenothiazine derivatives as pharmacotherapeutics for prion disease. Proc. Natl. Acad. Sci. USA 98:9836-9841.

\section{0}

\section{TSE-diagnostic by fluorescence-correlation-spectroscop y}

O. Schäfer, E. Birkmann, D. Riesner

University of Düsseldorf (Düsseldorf, D)

A characteristic feature of transmissible spongiform encephalopathies is the accumulation of a pathological isoform of the host encoded prion protein, PrP. The cellular isoform is designated $\mathrm{PrP}^{\wedge} \mathrm{C}$ and the pathological one $\mathrm{PrP}^{\wedge} \mathrm{Sc}$, respectively. PrP^Sc forms aggregates and is partially resistant to protease digestion. These properties distinguish $\mathrm{PrP}^{\wedge} \mathrm{Sc}$ from $\mathrm{PrP}^{\wedge} \mathrm{C}$ as a disease-specific marker in the central nervous system and peripheral tissues of infected animal and are used in all tests commercially available so far.

We present a sensitive biophysical test system based on Fluorescence-Correlation-Spectroscop y (FCS), which detects and quantifies aggregated proteins in solution. Single protein aggregates are fluorescence labelled with specific antibodies by FCS and can be detected as single particles. The principle was already successfully applied to the detection of the Abeta-peptide and PrP-aggregates in cerebrospinal fluid of Alzheimer disease patients (1) and CJD patients (2) respectively.
The sensitivity of the test was enhanced by improving the experimental setup and the sample processing. This system was applied to nervous tissue of scrapie infected hamsters. Animals in the progressed stage of the disease could be distinguished from a control group with $100 \%$ specificity and further experiments determining the sensitivity are in progress. For BSE we improved the sample processing (3) to avoided proteinase $\mathrm{K}$ digestion which otherwise limits the sensitivity.

1. Pitschke, et al. (1998). Nature Med 4: 832-834

2. Bieschke, et al. (2000). PNAS 97: 5468-5473

\section{1}

\section{Epstein Barr virus and cancer}

N. Raab-Traub

University of North Carolina (Chapel Hill, USA)

The Epstein-Barr virus (EBV) contributes to the development of several human cancers, including post-transplant lymphoma, a subset of AIDs related lymphomas, Hodgkin's lymphoma, and nasopharyngeal carcinoma (NPC). These malignancies are clonal proliferations that develop from an EBV-infected cell. Viral expression differs in the various pathologies associated with EBV, however, in most of the malignancies, the viral genes expressed include the EBV latent membrane proteins 1 and 2 (LMP1 \& 2), the EBV nuclear antigen 1, the EBER transcripts, and a family of RNAs encoded by the BamHI A EBV fragment.

LMP1 has transforming properties in rodent fibroblasts and is essential for EBV induced transformation of lymphocytes. It is expressed in NPC and in rare, early neoplastic nasopharyngeal lesions. LMP1 interacts with the signaling molecules for the tumor necrosis factor family of receptors (TRAFS) and is considered a constitutively activated member of this receptor family. This interaction activates the NFkB transcription factor and results in profound effects on cellular gene expression. Transgenic mice that express LMP1 under the control of an immunoglobulin heavy chain promoter and enhancer develop lymphoma with 3-4 times increased incidence than that of age-matched negative controls. These data indicate that LMP1, without expression of other EBV genes, is oncogenic in vivo and suggest that LMP1 is a major contributing factor to the development of EBVassociated lymphomas. To evaluate genetic factors that may contribute to oncogenesis, the LMP1 transgenic mice have been crossed with genetically engineered mice to evaluate the potential loss of the p16 tumor suppressor gene. The effects on tumorigenesis will be presented.

A second gene expressed in EBV associated tumors is LMP2 which is not essential for B cell transformation. In epithelial cell lines grown in vitro, LMP2 induces proliferation and inhibits differentiation with activation of the serinethreonine kinase, Akt. In vivo, activated Akt was detected in rare Reed-Steinberg cells in EBV negative lymphomas but was readily detected in most of the Reed-Steinberg cells in EBV positive samples and in the C15 NPC tumor. These data suggest that LMP2 is expressed in HD and NPC and that one consequence of this expression is activation of the Akt kinase. The consistent expression of multiple viral proteins in EBV associated malignancies provides new opportunities for anti-viral therapies targeted to viral:cell protein interactions and virally activated signaling pathways. 
112

\section{IGF-I receptor signaling system in JCV} T-antigen-induced primitive neuroectodermal tumors-medulloblastomas

A. Lassak, L. Del Valle, F. Peruzzi, J. Wang, S. Croul, K. Khalili, K. Reiss

Temple University (Philadelphia, USA)

Human neurotropic polyomavirus JC, considered the major etiologic factor in progressive multifocal leukoencephalopa thy (PML), has been detected in a number of cancers including cerebellar tumors of the childhood-medulloblastomas. Transforming potential of the early viral protein, JC virus large T-antigen, is well documented in a variety of cell lines and in experimental animals. Although the pathway/s by which T-antigen induces uncontrolled cell growth are not fully defined, T-antigen-mediated inactivation of tumor suppressor proteins represents one possible mechanism. Here we propose a novel functional interaction between JCV T-antigen and the IGF-I receptor signaling system. It involves a direct interaction with cytoplasmic protein-insulin receptor substrate 1 (IRS-1) and translocation of the T-antigen-IRS-1 complex into the nucleus. We report for the first time immunocytochemical detection of nuclear IRS-1 in JCV T-antigen positive murine medulloblastoma cell lines, in fibroblasts stably transfected with JCV T-antigen cDNA, and in living cells expressing a GFP-IRS-1 fusion protein. Importantly, IRS-1 co-precipitates with the JCV T-antigen and the interaction is independent of IRS-1 tyrosine phosphorylation and is strongly inhibited by IRS-1 serine phosphorylation. We further show that the IRS-1-JCV T-antigen binding is direct, it maps within the N-terminal portion of IRS-1, and that the N-terminal fragment (PH/PTB) efficiently competes for the T-antigen binding, and inhibits anchorageindependent growth of JCV T-antigen positive medulloblastoma cell lines. Importantly, nuclear IRS-1 was detected in human medulloblastoma biopsies positive for anti JCV T-antigen immunostaining. Based on these findings, we postulate that the interaction with T-antigen may uncouple IRS-1 from its surface receptors initiating series of nuclear events, which in turn may affect JCV T-antigen-mediated cellular transformation. 